\title{
Uso de la matriz dérmica acelular para el tratamiento de zonas críticas en defectos de cobertura. Serie de casos
}

\author{
Mariano O. Abrego, Javier Sánchez Saba, Ezequiel E. Zaidenberg, Ignacio Rellán, Agustín Donndorff, \\ Gerardo Gallucci, Pablo De Carli, Jorge G. Boretto \\ Sector de Cirugía de Mano y Miembro Superior, Instituto de Ortopedia y Traumatología "Prof. Dr. Carlos E. Ottolenghi”, \\ Hospital Italiano de Buenos Aires, Ciudad Autónoma de Buenos Aires, Argentina
}

\begin{abstract}
RESUMEN
Introducción: El objetivo de esta serie de casos es describir los resultados y las complicaciones de pacientes con heridas graves con defecto de cobertura en zonas críticas tratadas con una matriz dérmica acelular. Materiales y Métodos: Se realizó una revisión retrospectiva de los pacientes con déficit de cobertura en zonas críticas tratados con matriz dérmica acelular en nuestro centro. Definimos como zona crítica al déficit de cobertura que no pueda ser tratado solo con injerto de piel. Evaluamos variables preoperatorias, intraoperatorias y posoperatorias. Resultados: Los diagnósticos iniciales fueron tumor (3 pacientes), trauma agudo (3 pacientes) y secuela de síndrome compartimental (2 pacientes). El defecto de cobertura estaba localizado en la pierna (3 casos), en la mano ( 2 casos), en el muslo ( 1 caso), en el antebrazo ( 1 caso) y en el pie (1 caso). La zona crítica se caracterizó por exposición tendinosa con pérdida de peritenon ( 5 casos), exposición ósea y pérdida de periostio (1 caso), exposición de injerto de nervio (1 caso) y exposición de osteosíntesis (1 caso). Se registraron tres complicaciones. Dos pacientes requirieron una nueva colocación de matriz y, en otro paciente, se realizó un colgajo neurocutáneo de safeno interno por fracaso de la cobertura con membrana. Conclusiones: Los sustitutos dérmicos se caracterizan por su fácil uso y versatilidad. Esta técnica otorga protección en situaciones de exposición ósea, además de proveer un plano de deslizamiento en caso de exposición tendinosa. El uso de matriz dérmica permite, además, ahorrar el empleo de colgajos.
\end{abstract}

Palabras clave: Sustituto dérmico; matriz dérmica acelular; defecto de cobertura; cirugía reconstructiva.

Nivel de Evidencia: IV

The Role of Dermal Regeneration Templates in Severe Soft Tissue Defects. Management of "Critical Zones". Case Series

\begin{abstract}
Introduction: The aim of this study is to report outcomes and complications in a series of patients suffering soft tissue defects in critical areas treated using an acellular dermal matrix. Materials and Methods: A retrospective review of patients with soft tissue coverage deficits in critical areas treated with acellular dermal matrix in our center over a five-year period was conducted. The preoperative variables analyzed were: age, sex, comorbidities, number of previous surgeries, size and characteristics of the critical area and cause of the coverage defect. The intraoperative variables analyzed were: size of the dermal substitute used, surgical time and complications. The postoperative variables were: incorporation of the dermal substitute, time elapsed until the placement of the skin graft, and postoperative complications. Results: The initial diagnoses were tumor (3 patients), acute trauma (3 patients) and sequelae of compartment syndrome ( 2 patients). The soft tissue defect was located in the leg ( 3 cases), in the hand ( 2 cases), in the thigh ( 1 case), in the forearm ( 1 case) and in the foot ( 1 case). In 5 cases the critical zone was characterized by tendon exposure with loss of peritenon; in one case bone exposure and loss of periosteum; in one case exposure of nerve graft and in one case exposure of osteosynthesis material. Three complications were recorded. Conclusion: Dermal substitutes are characterized by their versatility. This technique can provide protection in situations of bone exposure, in addition to providing a sliding plane in case of tendon exposure. In situations of failure or impossibility of performing a biological reconstruction, dermal substitutes have a role within reconstructive options.
\end{abstract}

Key words: Dermal substitute; acelullar dermal matrix; soft tissue coverage; reconstructive surgery.

Level of Evidence: IV

Recibido el 14-5-2020. Aceptado luego de la evaluación el 9-9-2020 • Dr. MARIANO O. ABREGO • mariano.abrego@ hospitalitaliano.org.ar (ID https://orcid.org/0000-0001-9783-7373 Cómo citar este artículo: Abrego MO, Sánchez Saba J, Zaidenberg EE, Rellán I, Donndorff A, Gallucci G, De Carli P, Boretto JG. Uso de la matriz dérmica acelular para el tratamiento de zonas críticas en defectos de cobertura. Serie de casos. Rev Asoc Argent Ortop Traumatol 2021;86(2):167-174. https://doi.org/10.15417/issn.1852-7434.2021.86.2.1117 


\section{INTRODUCCIÓN}

La reconstrucción quirúrgica de los defectos de cobertura continúa siendo un desafío para el cirujano ortopédico. En ciertas situaciones, el empleo de colgajos o de injertos de piel no representa la solución al problema, ya sea por una contraindicación o una imposibilidad técnica. Bajo estas circunstancias es que los sustitutos dérmicos han ganado terreno, convirtiéndose en un recurso dentro del arsenal terapéutico del cirujano.

La matriz dérmica acelular es un tipo de sustituto cutáneo sintético compuesto principalmente por colágeno bovino y condroitín sulfato. ${ }^{1}$ Presenta una doble capa de matriz que estimula el crecimiento fibroblástico y endotelial mediante un proceso de imbibición, neovascularización y remodelado. ${ }^{2}$ Descrito originalmente por Burke y cols., el uso de matriz dérmica acelular para el desarrollo de la neodermis ha sido extensamente estudiado en los déficits de cobertura en quemaduras de espesor completo.

Con el paso del tiempo, la indicación para el empleo de matriz dérmica acelular se ha ampliado. ${ }^{3}$ En los últimos años, se han comunicado resultados satisfactorios con el uso de sustitutos dérmicos en heridas graves secundarias a un trauma, tanto en la enfermedad aguda como en defectos de cobertura crónicos. ${ }^{4-7}$ El objetivo de esta serie continua de casos es describir los resultados y las complicaciones en pacientes con heridas graves con defecto de cobertura en zonas críticas tratadas mediante el empleo de una matriz dérmica acelular.

\section{MATERIALES Y MÉTODOS}

Se llevó a cabo una revisión retrospectiva de los pacientes con déficit de cobertura de partes blandas tratados con matriz dérmica acelular en nuestro centro, por un mismo cirujano (JB), durante un período de cinco años (2014-2019). La información de los pacientes se extrajo del registro electrónico de historias clínicas de nuestra institución.

Se incluyó a pacientes, sin importar el sexo o la edad, con defecto de cobertura del miembro inferior o superior en "zonas críticas" tratados mediante la colocación de una matriz dérmica acelular Integra ${ }^{\circledR}$ (Integra Life Sciences Corporation, Plainsboro, NJ, EE.UU.) y la posterior reconstrucción con injerto de piel. Definimos como zona crítica al déficit de cobertura que no pueda ser tratado solo con injerto de piel, como la exposición ósea sin periostio o la exposición tendinosa sin peritenon. Se excluyó a los pacientes en quienes el sustituto dérmico se había utilizado en zonas no críticas.

Se analizaron variables preoperatorias, intraoperatorias y posoperatorias. Las variables preoperatorias analizadas fueron: edad, sexo, comorbilidades, cantidad de cirugías previas, tamaño y características de la zona crítica y causa del defecto de cobertura. Las variables intraoperatorias analizadas fueron: tamaño del sustituto dérmico utilizado y complicaciones. Las variables posoperatorias fueron: incorporación del sustituto dérmico, tiempo transcurrido hasta la colocación del injerto de piel y complicaciones. Además, describimos la técnica quirúrgica para la colocación del sustituto dérmico y las etapas evolutivas.

\section{Téenica quirúrgica}

En un primer tiempo quirúrgico, el objetivo es colocar el sustituto dérmico en la zona del defecto. La medición del defecto es fundamental para la planificación preoperatoria y así solicitar el tamaño de sustituto dérmico adecuado.

Todos los procedimientos se llevaron a cabo en la sala de operaciones y bajo técnica estéril, previa asepsia y antisepsia. El desbridamiento de la herida es fundamental antes de colocar el sustituto dérmico. Es esencial realizar una minuciosa hemostasia del lecho antes de colocar la matriz dérmica. Esto favorece el contacto de la membrana con el lecho y reduce la formación de hematomas.

La bicapa de colágeno se coloca sobre el lecho, fijándola en sus bordes, ya sea con agrafes o puntos no reabsorbibles. Se realizan curaciones periódicas que dependen del débito de la herida o del uso de un sistema de aspiración negativa por un lapso promedio de tres semanas. Si se coloca un sistema de aspiración continua a presión negativa (SACPN), las curaciones incluyen el recambio de manera estéril, en quirófano, con cada curación, cada una semana. En este período, comienza la regeneración vascular en el lecho. A los siete días, el color de la membrana cambia debido a la infiltración celular. A los 14 días, la matriz se vuelve anaranjada, lo que indica neovascularización.

Aproximadamente a las tres semanas, la neodermis alcanza su grado de revascularización adecuado. Estas bicapas sintéticas presentan una cobertura removible de silicona, la cual debe ser extraída para colocar el injerto de piel de espesor parcial. 


\section{RESULTADOS}

Se identificaron 11 pacientes en los cuales se utilizó un sustituto dérmico para el manejo de defectos de cobertura durante el período estudiado (Tabla). Ocho pacientes cumplieron con los criterios de inclusión (2 mujeres y 6 hombres). Todos los pacientes incluidos en este estudio tenían cirugías previas por su enfermedad de base (tumores) o en el contexto de cirugías de urgencia (trauma, síndrome compartimental).

Tabla. Características de la población

\begin{tabular}{|c|c|c|c|c|c|c|c|c|c|c|c|}
\hline Caso & $\begin{array}{l}\text { Edad } \\
\text { (años) }\end{array}$ & $\begin{array}{l}\text { Comorbi- } \\
\text { lidades }\end{array}$ & $\begin{array}{l}\text { Seguimiento } \\
\text { (meses) }\end{array}$ & Enfermedad & $\begin{array}{l}\text { Causa } \\
\text { del } \\
\text { defecto }\end{array}$ & $\begin{array}{l}\text { Locali- } \\
\text { zación }\end{array}$ & $\begin{array}{c}\text { Procedimientos } \\
\text { previos }\end{array}$ & $\begin{array}{l}\text { Tamaño } \\
\text { del } \\
\text { defecto } \\
(\mathrm{cm})\end{array}$ & $\begin{array}{l}\text { Caracte- } \\
\text { rísticas del } \\
\text { defecto }\end{array}$ & $\begin{array}{c}\text { Compli- } \\
\text { caciones } \\
\text { del } \\
\text { sustituto } \\
\text { dérmico }\end{array}$ & $\begin{array}{l}\text { Trata- } \\
\text { miento } \\
\text { de la } \\
\text { compli- } \\
\text { cación }\end{array}$ \\
\hline 1 & 38 & $\begin{array}{l}\text { Taba- } \\
\text { quismo }\end{array}$ & 22 & $\begin{array}{l}\text { Aplasia } \\
\text { medular } \\
\text { severa }\end{array}$ & $\begin{array}{l}\text { Síndrome } \\
\text { compar- } \\
\text { timental }\end{array}$ & Mano & $\begin{array}{c}\text { Fasciotomía/ } \\
\text { CAV/ } \\
\text { lipotransferencia }\end{array}$ & $9 \times 10$ & $\begin{array}{l}\text { Exposición } \\
\text { tendinosa }\end{array}$ & No & - \\
\hline 2 & 41 & No & 51 & $\begin{array}{l}\text { Mixofibrosar- } \\
\text { coma de alto } \\
\text { grado }\end{array}$ & $\begin{array}{l}\text { Resección } \\
\text { oncológica }\end{array}$ & Pierna & $\begin{array}{l}\text { Colgajo de dorsal } \\
\text { ancho }\end{array}$ & $20 \times 25$ & $\begin{array}{c}\text { Exposición } \\
\text { tendinosa }\end{array}$ & Sí & $\begin{array}{l}\text { Recolo- } \\
\text { cación de } \\
\text { membrana } \\
\text { Integra } \circledR\end{array}$ \\
\hline 3 & 38 & No & 16 & $\begin{array}{l}\text { Condrosarcoma } \\
\text { grado II }\end{array}$ & $\begin{array}{l}\text { Resección } \\
\text { oncológica }\end{array}$ & Pierna & $\begin{array}{l}\text { Aloprótesis de } \\
\text { resección tumoral }\end{array}$ & $2 \times 2$ & $\begin{array}{c}\text { Exposición } \\
\text { de material de } \\
\text { osteosíntesis }\end{array}$ & Sí & $\begin{array}{l}\text { Colgajo } \\
\text { neurocu- } \\
\text { táneo de } \\
\text { safeno } \\
\text { interno }\end{array}$ \\
\hline 4 & 62 & $\begin{array}{c}\text { Tabaquismo/ } \\
\text { diabetes } \\
\text { tipo } 2\end{array}$ & 20 & Trauma & $\begin{array}{l}\text { Síndrome } \\
\text { compar- } \\
\text { timental }\end{array}$ & Antebrazo & Fasciotomía/CAV & $10 \times 20$ & $\begin{array}{l}\text { Exposición } \\
\text { tendinosa }\end{array}$ & No & - \\
\hline 5 & 85 & No & 18 & Trauma & $\begin{array}{l}\text { Desguanta- } \\
\text { miento de pie }\end{array}$ & Pie & CAV & $10 \times 20$ & $\begin{array}{l}\text { Exposición } \\
\text { ósea }\end{array}$ & No & - \\
\hline 6 & 4 & No & 36 & Trauma & $\begin{array}{l}\text { Herida grave } \\
\text { con lesión } \\
\text { arterial }\end{array}$ & Muslo & $\begin{array}{l}\text { Colgajo de dorsal } \\
\text { ancho }\end{array}$ & $10 \times 25$ & $\begin{array}{l}\text { Exposición } \\
\text { nerviosa }\end{array}$ & No & - \\
\hline 7 & 35 & No & 38 & $\begin{array}{c}\text { Sarcoma sino- } \\
\text { vial monofásico }\end{array}$ & $\begin{array}{l}\text { Resección } \\
\text { oncológica }\end{array}$ & Pierna & CAV & $18 \times 12$ & $\begin{array}{l}\text { Exposición } \\
\text { tendinosa }\end{array}$ & Sí & $\begin{array}{l}\text { Recolo- } \\
\text { cación de } \\
\text { membrana } \\
\text { Integra } ₫\end{array}$ \\
\hline 8 & 58 & Tabaquismo & 8 & Trauma & $\begin{array}{l}\text { Fractura } \\
\text { expuesta }\end{array}$ & Mano & $\begin{array}{l}\text { Reducción y } \\
\text { osteosíntesis/ } \\
\text { colgajo de nervio } \\
\text { interóseo } \\
\text { posterior }\end{array}$ & $10 \times 20$ & $\begin{array}{l}\text { Exposición } \\
\text { tendinosa }\end{array}$ & No & - \\
\hline
\end{tabular}

$\mathrm{CAV}=$ cierre asistido por vacío

Tres pacientes tenían un diagnóstico tumoral como cuadro de base: dos con diagnóstico histológico de sarcoma de partes blandas (pierna y muslo) y uno con diagnóstico histológico de condrosarcoma (grado II, pierna). Los dos pacientes con sarcoma de partes blandas presentaban exposición tendinosa con ausencia de peritenon: uno evolucionó con un defecto de cobertura secundario a la resección oncológica, mientras que el otro evolucionó con un defecto de cobertura consecuencia de la resección oncológica y la posterior pérdida del colgajo de dorsal ancho y paraescapular. El tercer paciente (condrosarcoma) tenía una exposición del material de osteosíntesis secundaria a la cirugía de reconstrucción tumoral. 
Tres pacientes de nuestra serie tenían un defecto de cobertura secundario a un trauma agudo. Uno sufrió un desguantamiento del pie con exposición ósea y ausencia de periostio. Otro presentaba una herida con defecto de cobertura en el muslo con exposición tendinosa sin peritenon y, además, exposición del nervio ciático. En primera instancia, se realizó un colgajo de dorsal ancho, que evolucionó a una necrosis total. El tercer paciente tenía múltiples fracturas expuestas en la mano, con exposición tendinosa y ausencia de peritenon.

Los dos pacientes restantes presentaban un déficit de cobertura como consecuencia de un síndrome compartimental. El primero tenía una infección del catéter en el contexto de una aplasia medular severa, desarrolló un síndrome compartimental de la mano, tratado con fasciotomías (exposición ósea y necrosis del aparato extensor de los dedos en zona 6) (Figuras 1 y 2). El segundo desarrolló un síndrome compartimental del antebrazo como consecuencia de una fractura cerrada en el contexto de un politrauma, fue tratado con fasciotomías, evolucionó con déficit de cobertura por necrosis cutánea y exposición tendinosa con ausencia de peritenon. Cabe aclarar que, en este paciente, pese a haber sufrido un trauma, la causa del defecto de cobertura fue el resultado del tratamiento quirúrgico del síndrome compartimental.
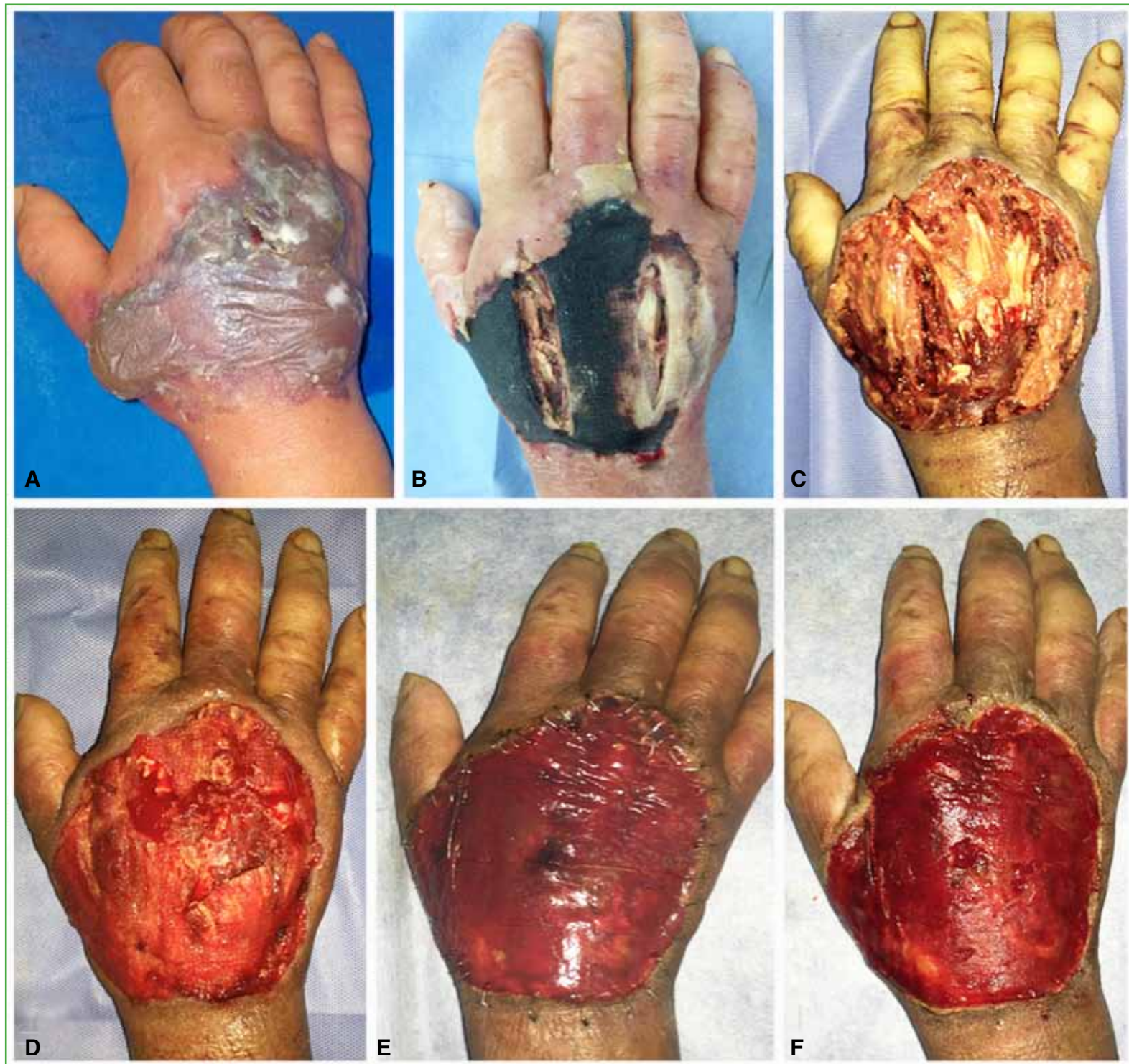

Figura 1. Caso 1. A. Síndrome compartimental secundario a infección por catéter. B. Evolución posfasciotomías.

C. Exposición tendinosa. D. Exposición ósea posterior a la necrosis de los tendones. E. Colocación de sustituto dérmico.

F. Matriz regenerada. 


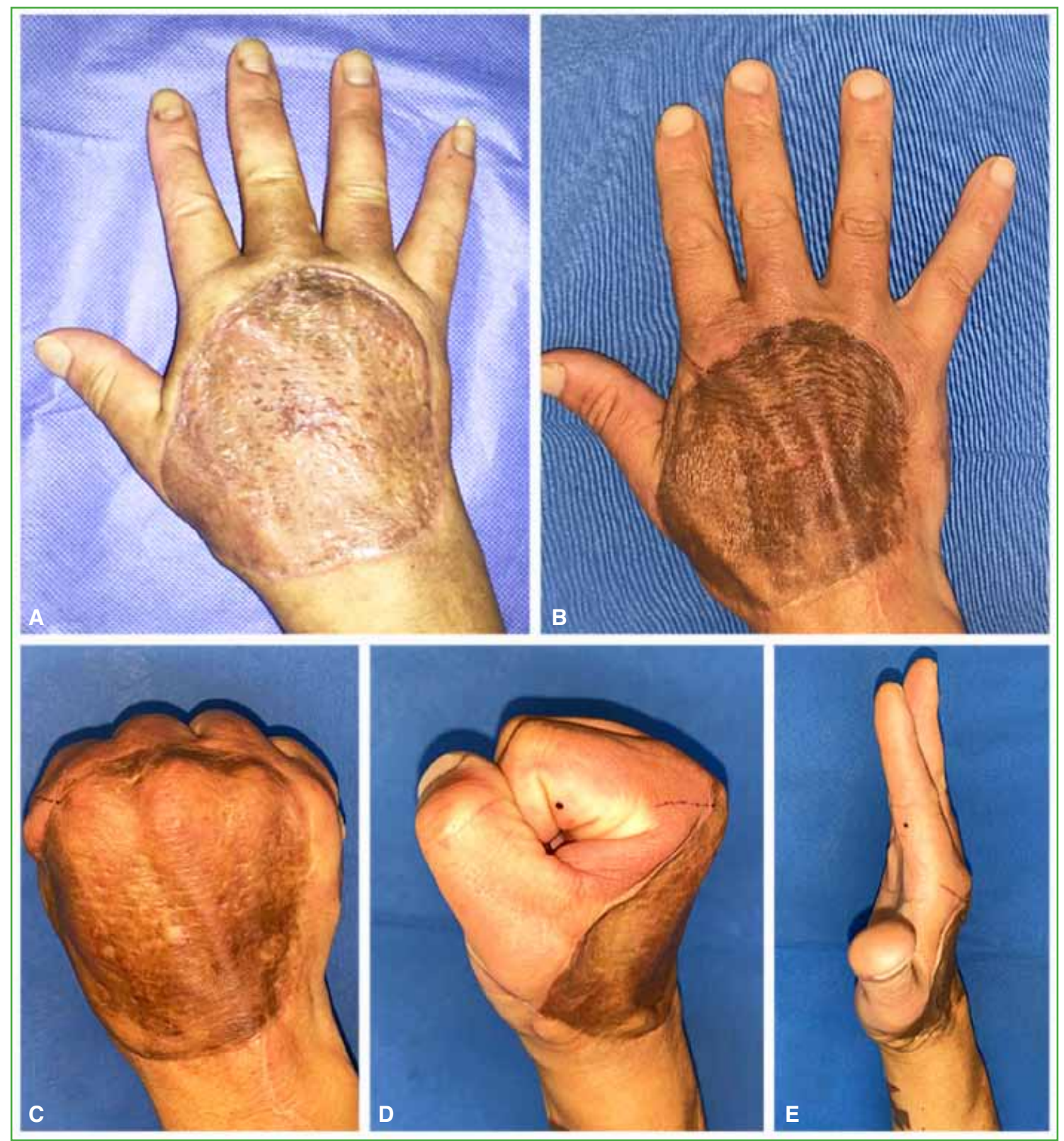

Figura 2. Caso 1. A. Colocación de injerto libre de piel. Seguimiento temprano. B-E. Seguimiento a largo plazo, después de la reconstrucción tendinosa.

El promedio de edad en el momento de la cirugía fue de 45 años (rango 4-85). El seguimiento promedio fue de 26 meses (rango 6-51). Como antecedentes preoperatorios de relevancia, se registraron tres pacientes tabaquistas, uno de ellos también sufría diabetes mellitus tipo 2. El tamaño promedio del sustituto dérmico fue de $140 \mathrm{~cm}^{2}$ (rango 4-250).

No se registraron complicaciones intraoperatorias. Hubo tres complicaciones posoperatorias. Dos pacientes requirieron una nueva colocación de matriz en la zona del defecto (sarcoma de partes blancas) y, en otro paciente (condrosarcoma grado II), se realizó un colgajo neurocutáneo de safeno interno por fracaso de la cobertura con membrana. 


\section{DISCUSIÓN}

Este estudio comunica el uso de una matriz dérmica acelular y la colocación de injerto libre de piel en zonas críticas en defectos de cobertura, en ocho pacientes. En la evaluación final, el resultado fue satisfactorio en siete de los ocho pacientes.

En el contexto de una herida grave con déficit de cobertura, los objetivos terapéuticos básicos son: remover la contaminación, identificar y clasificar la gravedad de la lesión, el control hemostático, el desbridamiento, la estabilización ósea, el restablecimiento de la circulación (reparación directa o injerto), la reparación nerviosa, la reparación tendinosa y la cobertura de partes blandas. ${ }^{8}$

El proceso de cicatrización de heridas involucra una cascada multicelular (queratinocitos, células endoteliales, macrófagos, plaquetas, fibroblastos) que llevan a la respuesta inflamatoria y la posterior formación de tejido nuevo. ${ }^{9}$

Los sustitutos dérmicos de tipo acelulares se originan de material dérmico cadavérico, el cual es sometido a un proceso de remoción celular y de material antigénico, como así también de material infeccioso. ${ }^{10,11}$

Los sustitutos dérmicos se caracterizan por su fácil uso y versatilidad. Basándose en el grado de complejidad del procedimiento, la escalera reconstructiva modificada por Rehim y cols. ${ }^{12}$ posiciona a los sustitutos dérmicos entre los colgajos locales y los colgajos pediculados a distancia. Se han postulado las características del sustituto dérmico ideal, que incluyen: resistencia a la infección, ausencia de respuesta antigénica, perdurabilidad, fácil acceso, estable y con capacidad de cubrir un amplio espectro de heridas. ${ }^{13}$ En los últimos años, la evidencia médica sobre el uso de sustitutos dérmicos en úlceras venosas y pacientes diabéticos ha aumentado considerablemente. ${ }^{14,15}$

En ciertas ocasiones, no es posible tratar la exposición de estructuras profundas (tendones, huesos, nervios, material de osteosíntesis) con simples injertos de piel. ${ }^{16}$ Además, no siempre se dispone de la posibilidad de confeccionar colgajos, ya sea por comorbilidad clínica del paciente o antecedentes quirúrgicos que lo contraindiquen. Los sustitutos dérmicos tienen la capacidad de proveer un plano de deslizamiento adecuado en caso de exposición tendinosa. Sin embargo, la calidad biológica de la reconstrucción con un sustituto dérmico es inferior a la de los colgajos.

En nuestra serie, hubo tres complicaciones. En dos pacientes, se decidió recolocar la membrana, ambos casos con resultados satisfactorios. El tercer paciente tenía un contexto particular, por el cual no recomendamos realizar un nuevo intento con matriz dérmica. Como bien sabemos la neovascularización de la membrana biológica se realiza desde la periferia cuando el lecho es avascular o hipovascular. En este caso, la vitalidad de los bordes del defecto de cobertura estaba alterada debido a la radioterapia. Además, el material de osteosíntesis de esta paciente estaba expuesto y fijado a un aloinjerto óseo. Todo esto determina que la indicación de matriz dérmica no sea la adecuada en caso de fracaso en primera instancia. Creemos que las características particulares del defecto (bordes sufridos por radioterapia, hueso de banco y osteosíntesis expuesta) son demasiado desfavorables para insistir con un tratamiento poco invasivo, como la colocación de un sustituto dérmico. Sin embargo, no podemos afirmar si la exposición de la osteosíntesis per se es una contraindicación.

Una de las desventajas de los sustitutos dérmicos es el alto costo. ${ }^{17}$ Además, si bien hay reportes de colocación de Integra ${ }^{\circledR}$ y el posterior injerto de piel en un solo acto quirúrgico, este procedimiento suele realizarse en dos tiempos y esto implica dos procedimientos, lo que incrementa aún más los costos. ${ }^{18}$ Se ha descrito la formación de seromas en el posoperatorio; esto puede controlarse perforando la membrana y permitiendo así el drenaje de fluido. ${ }^{19}$

Si bien la colocación de SACPN no es una indicación obligatoria para el uso de sustitutos dérmicos, se ha comprobado que aumentan la velocidad de vascularización de la membrana ${ }^{20}$ Los SACPN tienden a acelerar el proceso de neoformación tisular. En estudios previos, la tasa de éxito con SACPN fue del $90 \%$ en comparación con el uso de membrana sin SACPN (75\%). Además, el uso de SACPN daría mayor estabilidad y contacto entre la membrana y el lecho. Por otro lado, el SACPN eliminaría el exceso de secreción exudativa y, por ende, reduce, al mínimo, la eventual proliferación de bacterias, teniendo en cuenta que los sustitutos dérmicos tienden a ser colonizados con facilidad. ${ }^{16,21}$ Sin embargo, el empleo de SACPN implica un costo extra, porque los recambios se deben realizar en el quirófano, lo que aumenta el costo del tratamiento. Sin embargo, las curaciones se efectúan cada siete días cuando se emplea el SACPN y, entre las 24 y $72 \mathrm{~h}$, cuando no se lo utiliza. Si es posible, recomendamos usar SACPN, teniendo en cuenta el costo extra y la logística que implica. Aun así, los resultados sin SACPN siguen siendo satisfactorios. Luego de colocar el injerto de piel, se vuelve a colocar la terapia de presión negativa para obtener una incorporación más rápida y completa del injerto.

En coincidencia con la bibliografía, creemos que, a medida que se amplíen las indicaciones para el uso de sustitutos dérmicos, crecerá la necesidad de estudios comparativos prospectivos para evaluar los beneficios de la técnica a largo plazo..$^{12,22,23}$ 
La limitación más importante de este estudio es su característica retrospectiva, sumada a la baja cantidad de pacientes. Sin embargo, en la bibliografía internacional, no se definen, con exactitud, las zonas críticas mencionadas en nuestro estudio y las series suelen ser heterogéneas, incluyen defectos de cobertura sin exposición de estructuras nobles.

\section{CONCLUSIONES}

La combinación del uso de sustitutos dérmicos y la posterior colocación de injerto de piel representa una variante versátil y relativamente sencilla para el manejo de los defectos de cobertura. Además, esta técnica puede indicarse cuando el defecto de cobertura incluya zonas críticas (exposición tendinosa con ausencia de peritenon, exposición ósea sin periostio, estructuras neurovasculares expuestas) y el empleo directo de injerto de piel esté contraindicado. Los sustitutos dérmicos han de ser considerados una herramienta para la reconstrucción de defectos de cobertura en las extremidades.

Conflicto de intereses: Los autores no declaran conflictos de intereses.

ORCID de J. Sánchez Saba: https://orcid.org/0000-0001-5496-3513 ORCID de E. E. Zaidenberg: https://orcid.org/0000-0002-1535-0586 ORCID de I. Rellán: https://orcid.org/0000-0003-4045-339X ORCID de A. Donndorff: https://orcid.org/0000-0002-6384-4820
ORCID de G. Gallucci: https://orcid.org/0000-0002-0612-320X ORCID de P. De Carli: https://orcid.org/0000-0002-9474-8129 ORCID de J. G. Boretto: https://orcid.org/0000-0001-7701-3852

\section{BIBLIOGRAFÍA}

1. Burke JF, Yannas IV, Quinby WC, Bondoc CC, Jung WK. Successful use of a physiologically acceptable artificial skin in the treatment of extensive burn injury. Ann Surg 1981;194:413-28. https://doi.org/10.1097/00000658-198110000-00005

2. Moiemen NS, Staiano JJ, Ojeh NO, Thway Y, Frame JD. Reconstructive surgery with a dermal regeneration template: clinical and histologic study. Plast Reconstr Surg 2001;108:93-103. https://doi.org/10.1097/00006534-200107000-00015

3. Reynolds M, Kelly DA, Walker NJ, Crantford C, Defranzo AJ. Use of integra in the management of complex hand wounds from cancer resection and nonburn trauma. Hand (NY) 2018;13:74-9. https://doi.org/10.1177/1558944717692090

4. Shores JT, Hiersche M, Gabriel A, Gupta S. Tendon coverage using an artificial skin substitute. J Plast Reconstr Aesthet Surg 2012;65:1544-550. https://doi.org/10.1016/j.bjps.2012.05.021

5. Weigert R, Choughri H, Casoli V. Management of severe hand wounds with Integra ${ }^{\circledR}$ dermal regeneration template. J Hand Surg Eur 2011;36:185-93. https://doi.org/10.1177/1753193410387329

6. Dai C, Shih S, Khachemoune A. Skin substitutes for acute and chronic wound healing: an updated review. $J$ Dermatolog Treat 2020;31(6):639-48. https://doi.org/10.1080/09546634.2018.1530443

7. Leclère FM, Desnouveaux E, Choughri H, Casoli V. Acellular dermal matrix: New applications for free flap pedicle coverage - A prospective study in 10 patients. J Cosmet Laser Ther 2018;20:200-4. https://doi.org/10.1080/14764172.2016.1248439

8. Latifi R, El-Hennawy H, El-Menyar A, Peralta R, Asim M, Consunji R, et al. The therapeutic challenges of degloving soft-tissue injuries. J Emerg Trauma Shock 2014;7:228-32. https://doi.org/10.4103/0974-2700.136870

9. De Angelis B, Orlandi F, Fernandes Lopes Morais D’ Autilio M, Scioli MG, Orlandi A, Cervelli V, et al. Long-term follow-up comparison of two different bi-layer dermal substitutes in tissue regeneration: Clinical outcomes and histological findings. Int Wound J 2018;15:695-706. https://doi.org/10.1111/iwj.12912

10. Debels H, Hamdi M, Abberton K, Morrison W. Dermal matrices and bioengineered skin substitutes: a critical review of current options. Plast Reconstr Surg Glob Open 2015;3 e284. https://doi.org/10.1097/GOX.0000000000000219

11. Shores JT, Gabriel A, Gupta S. Skin substitutes and alternatives: a review. Adv Skin Wound Care 2007;20:493-508; quiz 509. https://doi.org/10.1097/01.ASW.0000288217.83128.f3 
12. Rehim SA, Singhal M, Chung KC. Dermal skin substitutes for upper limb reconstruction: current status, indications, and contraindications. Hand Clin 2014;30:239-52, vii. https://doi.org/10.1016/j.hcl.2014.02.001

13. Nathoo R, Howe N, Cohen G. Skin substitutes: an overview of the key players in wound management. J Clin Aesthet Dermatol 2014;7:44-8. PMID: 25371771

14. Winters CL, Brigido SA, Liden BA, Simmons M, Hartman JF, Wright ML. A multicenter study involving the use of a human acellular dermal regenerative tissue matrix for the treatment of diabetic lower extremity wounds. Adv Skin Wound Care 2008;21:375-81. https://doi.org/10.1097/01.ASW.0000323532.98003.26

15. Greaves NS, Iqbal SA, Baguneid M, Bayat A. The role of skin substitutes in the management of chronic cutaneous wounds. Wound Repair Regen 2013;21:194-210. https://doi.org/10.1111/wrr.12029

16. González Alaña I, Torrero López JV, Martín Playá P, Gabilondo Zubizarreta FJ. Combined use of negative pressure wound therapy and Integra ${ }^{\circledR}$ to treat complex defects in lower extremities after burns. Ann Burns Fire Disasters 2013;26:90-3. Disponible en: http://www.medbc.com/annals/review/vol_26/num_2/text/vol26n2p90.pdf

17. Bello YM, Falabella AF, Eaglstein WH. Tissue-engineered skin. Current status in wound healing. Am J Clin Dermatol 2001;2:305-13. https://doi.org/10.2165/00128071-200102050-00005

18. Demiri E, Papaconstantinou A, Dionyssiou D, Dionyssopoulos A, Kaidoglou K, Efstratiou I. Reconstruction of skin avulsion injuries of the upper extremity with Integra(®) dermal regeneration template and skin grafts in a singlestage procedure. Arch Orthop Trauma Surg 2013;133:1521-6. https://doi.org/10.1007/s00402-013-1834-2

19. Hanft JR, Surprenant MS. Healing of chronic foot ulcers in diabetic patients treated with a human fibroblast-derived dermis. J Foot Ankle Surg 2002;41:291-9. https://doi.org/10.1016/s1067-2516(02)80047-3

20. Hansbrough JF, Doré C, Hansbrough WB. Clinical trials of a living dermal tissue replacement placed beneath meshed, split-thickness skin grafts on excised burn wounds. J Burn Care Rehabil 1992;13:519-29. https://doi.org/10.1097/00004630-199209000-00004

21. Molnar JA, Defranzo AJ, Hadaegh A, Morykwas MJ, Shen P, Argenta LC. Acceleration of Integra incorporation in complex defects with subatmospheric pressure. Plast Reconstr Surg 2004;113:1339. https://doi.org/10.1097/01.prs.0000112746.67050.68

22. Sando IC, Chung KC. The use of dermal skin substitutes for the treatment of the burned hand. Hand Clin 2017;33:269-76. https://doi.org/10.1016/j.hcl.2016.12.008

23. Danin A, Georgesco G, Touze AL, Penaud A, Quignon R, Zakine G. Assessment of burned hands reconstructed with Integra(®) by ultrasonography and elastometry. Burns 2012;38:998-1004. https://doi.org/10.1016/j.burns.2012.02.017 